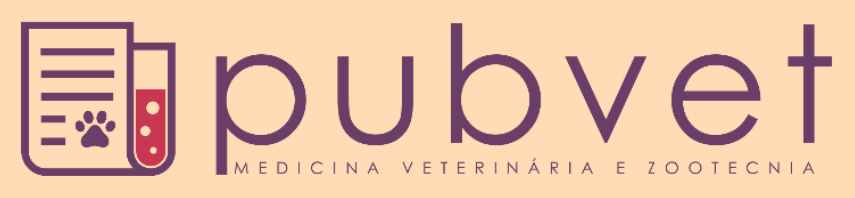

https://doi.org/10.31533/pubvet.v15n08a884.1-7

\title{
Vulvovaginite por estrutura óssea no canal vaginal de cadela castrada: Relato de caso
}

\author{
Gabriela Vanessa da Silva ${ }^{1 *}$, Suélen Dalegrave ${ }^{2}$, Eduardo Conceição de Oliveira $^{3}$ E $^{\circ}$, Laís \\ Rezzadori Flecke $^{4}$, Luana Baptista de Azevedo ${ }^{5}{ }^{\circ}$, Carolain Schorr Daga ${ }^{\circ}$, Elis Natacha \\ Wendpap $^{60}$, Maria Cecilia de Lima Rorig ${ }^{7}$
}

${ }^{1}$ Discente do curso de Medicina Veterinária da Pontifícia Universidade Católica do Paraná (PUCPR) Toledo, Paraná, Brasil. ${ }^{2}$ Aprimoranda do Programa de Aprimoramento de Clínica Médica e Cirúrgica de Pequenos Animais, PUCPR, Toledo, Brasil.

${ }^{3}$ Medico Veterinário, Docente do curso de Medicina Veterinária da Universidade de Caxias do Sul, Caxias do Sul, Rio Grande do Sul, Brasil. ${ }^{4}$ Aprimoranda do Programa de Clínica Médica de Pequenos Animais, Universidade de Caxias do Sul, Caxias do Sul, Rio Grande do Sul, Brasil. ${ }^{5}$ Médica veterinária, em diagnóstico por imagem, Caxias do Sul, Rio Grande do Sul, Brasil.

${ }^{6}$ Médica veterinária, em clínica médica de pequenos animais, Toledo, Paraná, Brasil.

${ }^{7}$ Médico(a) veterinário(a) docente do curso de Medicina Veterinária da PUCPR, Toledo, Paraná, Brasil.

*Autor para correspondência: gabivanessa97@outlook.com

Resumo. O sistema reprodutivo das fêmeas é considerado altamente importante para a sobrevivência da espécie. O objetivo deste trabalho é relatar o atendimento de uma cadela adulta castrada, com diagnóstico de estrutura óssea no canal vaginal, ocasionando vaginite e cistite recorrentes. A paciente chegou para atendimento com histórico de polaquiúria, secreção vulvar purulenta e lambedura excessiva da vulva há dois meses. No exame físico constatou-se mucosas normocoradas, frequência respiratória normal, sopro grau 3 à auscultação cardíaca e durante o toque vaginal apresentava bastante sensibilidade dolorosa. O diagnóstico foi realizado através de radiografia ventro dorsal de pelve, que revelou presença de três estruturas radiopacas no canal vaginal além do exame ultrassonográfico que evidenciou cistite. O tratamento se baseou na remoção manual das estruturas presentes, sob anestesia geral, com auxílio de um espéculo vaginal e pinça hemostática. Após o procedimento de remoção das estruturas ósseas, administrou-se na paciente por via intravenosa meloxicam $(0,1 \mathrm{mg} / \mathrm{kg})$; dipirona sódica $(25 \mathrm{mg} / \mathrm{kg})$ e enrofloxacina $(10 \mathrm{mg} / \mathrm{kg})$ por via subcutânea (SC). O material foi encaminhado para exame histopatológico em formol a $10 \%$. No exame histopatológico notou-se a presença de matriz óssea mineralizada, associada a estrutura tecidual com morfologia de pele e restos de folículos pilosos e neles queratina. A paciente recebeu alta após a recuperação anestésica com prescrição de enrofloxacina $10 \mathrm{mg} / \mathrm{kg} \mathrm{SID}$, durante sete dias; meloxicam $0,1 \mathrm{mg} / \mathrm{kg}$, SID, durante três dias e cloridrato de tramadol $4 \mathrm{mg} / \mathrm{kg}$ BID, durante cinco dias. Este trabalho demostra a importância do diagnóstico baseado nas informações alcançadas na anamnese, e no exame físico completo, considerando o exame ginecológico neste contexto, realizado pelo toque vaginal, além de outros mecanismos que são necessários para estabilização do paciente e correção da enfermidade, com propósito de obter melhora do quadro clínico e sucesso na terapêutica.

Palavras chave: Diagnóstico, estrutura radiopaca, radiografia, vaginite

\section{Bone structure vulvovaginitis in the vaginal canal of castrated canine female: Case report}

Abstract. The reproductive system of females is considered highly important for the survival of the species. The aim of this study is to report the care of a spayed adult female dog, diagnosed with bone structure in the vaginal canal, causing recurrent vaginitis and 
cystitis. The patient arrived for care with a history of polaquiuria, purulent vulvar discharge and excessive licking of the vulva 2 months ago. On physical examination, normal mucous membranes were found, normal respiratory rate, grade 3 murmur on cardiac auscultation, and during vaginal touch, she had a lot of painful sensitivity. The diagnosis was made by radiography of the dorsal pelvis, which revealed the presence of three radiopaque structures in the vaginal canal, in addition to an ultrasound examination that showed cystitis. The treatment was based on the manual removal of the present structures, under general anesthesia, with the aid of a vaginal speculum and hemostatic forceps. After the bone structure removal procedure, meloxicam $(0.1 \mathrm{mg} / \mathrm{kg})$ was administered to the patient intravenously; dipyrone sodium $(25 \mathrm{mg} / \mathrm{kg})$ and enrofloxacin (10 mg / kg) subcutaneously (SC). The material was sent for histopathological examination in $10 \%$ formaldehyde. Histopathological examination revealed the presence of mineralized bone matrix associated with tissue structure with skin morphology containing hair follicle remains and keratin in them. The patient was discharged after anesthetic recovery with a prescription for enrofloxacin $10 \mathrm{mg} / \mathrm{kg}$ SID for 7 days; Meloxicam $0.1 \mathrm{mg} / \mathrm{kg}$, SID, for 3 days and tramadol hydrochloride $4 \mathrm{mg} / \mathrm{kg}$ BID, for 5 days. This work demonstrates the importance of the diagnosis based on the information obtained in the anamnesis, and in the complete physical examination, considering the gynecological examination in this context, performed through vaginal touch, in addition to other mechanisms that are necessary for stabilizing the patient and correcting the disease, with purpose of improving the clinical picture and success in therapy.

Keywords: Diagnosis, radiopaque structure, radiography, vaginitis

\section{Vulvovaginitis con estructura ósea en el canal vaginal de una canina etserilizada: Reporte de caso}

Resumen. El sistema reproductivo de las hembras se considera muy importante para la supervivencia de la especie. El objetivo de este estudio es reportar el cuidado de un perro adulto castrado, diagnosticado con estructura ósea en el canal vaginal, causando vaginitis y cistitis recurrentes. La paciente llegó para recibir atención con antecedentes de polaquiuria, secreción vulvar purulenta y lamido excesivo de la vulva hace 2 meses. Al examen físico se encontró mucosas normales, frecuencia respiratoria normal, soplo grado 3 en la auscultación cardíaca, y durante el tacto vaginal tenía mucha sensibilidad dolorosa. El diagnóstico se realizó mediante radiografía de pelvis dorsal, que reveló la presencia de tres estructuras radiopacas en el canal vaginal, además de una ecografía que mostró cistitis. El tratamiento se basó en la remoción manual de las estructuras presentes, bajo anestesia general, con la ayuda de un espéculo vaginal y pinzas hemostáticas. Después del procedimiento de eliminación de la estructura ósea, se administró meloxicam $(0,1 \mathrm{mg} / \mathrm{kg})$ al paciente por vía intravenosa; dipirona sódica $(25 \mathrm{mg} / \mathrm{kg})$ y enrofloxacina $(10 \mathrm{mg} / \mathrm{kg}$ ) por vía subcutánea (SC). El material se envió para examen histopatológico en formaldehído al $10 \%$. En el examen histopatológico se apreció la presencia de matriz ósea mineralizada asociada con la estructura del tejido con morfología de la piel que contiene restos de folículos pilosos y queratina en ellos. El paciente fue dado de alta tras la recuperación anestésica con prescripción de enrofloxacino $10 \mathrm{mg} / \mathrm{kg}$ SID durante 7 días; Meloxicam 0,1 mg/kg, SID, durante 3 días e hidrocloruro de tramadol $4 \mathrm{mg} / \mathrm{kg}$ BID, durante 5 días. Este trabajo demuestra la importancia del diagnóstico a partir de la información obtenida en la anamnesis, y en el examen físico completo, considerando el examen ginecológico en este contexto, realizado a través del tacto vaginal, además de otros mecanismos que son necesarios para estabilizar a la paciente y corregir la enfermedad, con el fin de mejorar el cuadro clínico y el éxito de la terapia.

Palabras clave: Diagnóstico, estructura radiopaca, radiografía, vaginitis 


\section{Introdução}

O sistema reprodutivo das fêmeas é considerado altamente importante para a sobrevivência da espécie. Este é composto por ovários, tubas uterinas, útero, vagina e vulva, com objetivo promover a concepção, proporcionando um local adequado para a geração, crescimento e nutrição do feto no decorrer de toda gestação, além da produção de hormônios que controlam o ciclo estral (Zachary et al., 2012).

Segundo Nelson \& Couto (2015), distocia é a dificuldade da cadela gestante em expulsar o feto durante o trabalho de parto, possuindo um predomínio de $5 \%$ a $6 \%$ das gestações. Para Johnston et al. (2001) vários são os fatores para esta patologia entre elas deformidade do canal pélvico, tamanho do concepto, mal disposição fetal, ou conjunto de duas ou mais causas, visto que uma das consequências das distocia é a vaginite, que consiste em uma inflamação da vagina e do vestíbulo vaginal que pode ocorrer em qualquer idade, raça ou condição ovariana.

As causas de vaginite são diversas, sendo ordenada como primária nos casos de infecções causadas pelo herpes vírus canino tipo I (HVC I) e Brucella canis, e secundárias em decorrência de doenças do aparelho reprodutor, drogas imunossupressoras, ou antibióticos utilizados de forma profilática (Nelson \& Couto, 2015). Demais causas são capazes de estar relacionadas como infecção do trato urinário, incontinência urinária, infecções genitais, irritação mecânica motivada por corpos estranhos ou tumores (Purswell, 2004; Soderberg, 1986; Tilley et al., 2008).

Alterações reprodutivas na espécie canina são capazes de serem evidenciadas e apresentar efeitos variados, que podem dispor da inexistência ou poucos sinais clínicos, caracterizando desafios no diagnóstico e terapêutica, capaz de passar de forma despercebida ao tutor (Klein, 2007; Sapierzyński et al., 2007). Devido à escassez de estudos que descreva casos de estruturas ósseas em canal vaginal de cadela castrada, objetivou-se relatar o presente caso a fim de contribuir para epidemiologia da enfermidade.

\section{Relato de caso}

Foi atendida no Hospital Veterinário Aukmia, localizado em Toledo, Paraná uma cadela sem raça definida, adulta, 10 anos de idade, $6,6 \mathrm{~kg}$, castrada há cinco anos, com histórico de polaquiúria, secreção vulvar mucopurulenta e lambedura excessiva da vulva há dois meses. A paciente já havia sido atendida por outro profissional da área que prescreveu uso de antimicrobiano; porém, apresentou melhora seguida de recidiva.

Durante o exame físico foi verificado que a paciente se apresentava ativa, com mucosas normocoradas, frequência respiratória normal, e durante a auscultação cardíaca foi possível perceber existência de sopro grau três. No decorrer da palpação abdominal foi notado que o animal sentia desconforto, e bastante sensibilidade dolorosa ao toque vaginal. Diante das alterações constatadas no exame físico solicitou-se hemograma, perfil bioquímico, cultura bacteriana, antibiograma e ultrassonografia abdominal. Contudo, apenas o exame ultrassonográfico foi autorizado pelo tutor, e realizado para fins de diagnóstico.

No ultrassom abdominal (Figura 1) foram constatadas alterações compatíveis com cistite crônica. Prescreveu-se como terapêutica domiciliar omeprazol ( $1 \mathrm{mg} / \mathrm{kg} / \mathrm{SID})$ durante 10 dias; marbofloxacina $(5 \mathrm{mg} / \mathrm{kg} / \mathrm{SID})$, durante 10 dias; meloxicam $(0,1 \mathrm{mg} / \mathrm{kg} / \mathrm{SID})$, durante 5 dias; cloridrato de tramadol (4 $\mathrm{mg} / \mathrm{kg} / \mathrm{BID})$, durante 5 dias e dipirona sódica $(25 \mathrm{mg} / \mathrm{kg} / \mathrm{BID})$, durante 5 dias com orientação de retorno para reavaliação em 10 dias.

No retorno o tutor relatou que a paciente seguia com lambedura excessiva da vulva, no entanto a frequência da micção estava normal. No decorrer do exame físico o animal ainda sentia bastante dor durante a manipulação da vulva e cauda. Foi sugerido radiografia, que constatou a presença de estruturas radiopacas em canal vaginal (Figura 2). Em seguida, foi realizada inspeção vaginal com uso de espéculo, que possibilitou a visualização das estruturas presentes.

Diante dos achados radiográficos, e da visualização das estruturas pela inspeção vaginal procedeuse a remoção manual das mesmas, sob anestesia geral e com auxílio de um espéculo vaginal e pinça hemostática. Ao final do procedimento foram removidos três fragmentos ósseos (Figura 3). 
Após a recuperação anestésica a paciente recebeu alta com prescrição de enrofloxacina $(10 \mathrm{mg} / \mathrm{kg}$ /SID) durante cinco dias; meloxicam $(0,1 \mathrm{mg} / \mathrm{kg} / \mathrm{SID})$ durante três dias e cloridrato de tramadol quatro $\mathrm{mg} / \mathrm{kg} / \mathrm{BID}$ ) durante três dias, com indicação de retorno para reavaliação em sete dias.

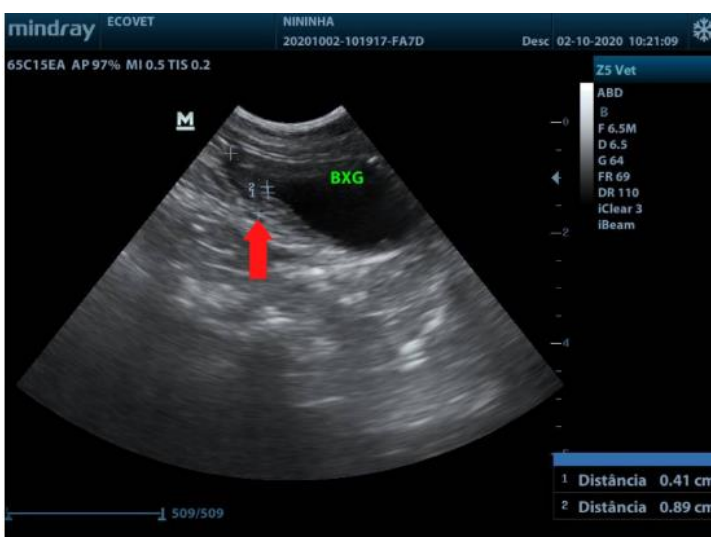

Figura 1. Vesícula urinária apresentando parede espessa e_hiperecogênica (flecha).
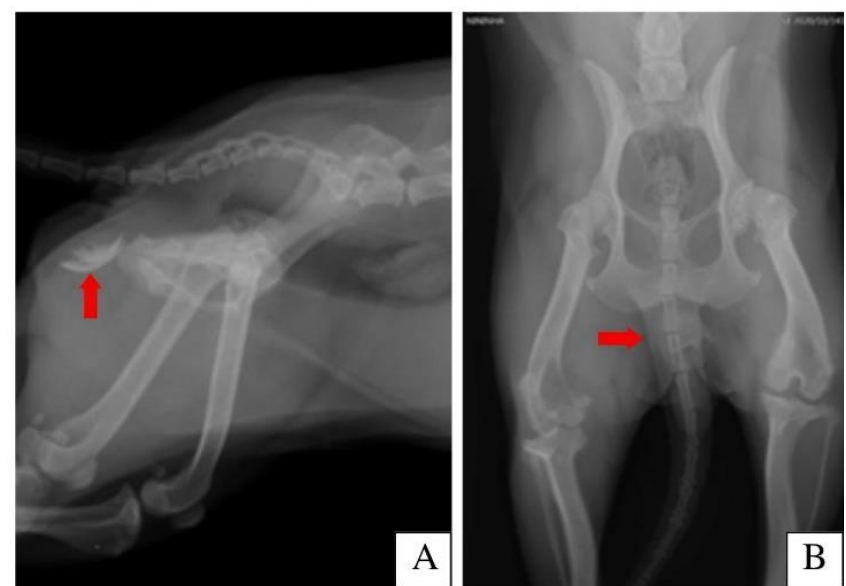

Figura 2. A. Radiografia laterolateral de pelve e membros pélvicos B. Radiografia ventrodorsal de pelve e membros pélvicos de cão: observa-se estrutura radiopaca em canal vaginal (flechas).

As estruturas removidas foram fixadas em formol tamponado a $10 \%$ e após fixação foi encaminhado para processo histopatológico. $\mathrm{O}$ exame histopatológico revelou presença de matriz óssea mineralizada associada a estrutura tecidual com morfologia de pele contendo restos de folículos pilosos e neles queratina (Figura 4).

No retorno após sete dias, o tutor relatou que a paciente estava bem clinicamente após a remoção das estruturas, com micção normal, sem secreção e lambedura da região vulvar.

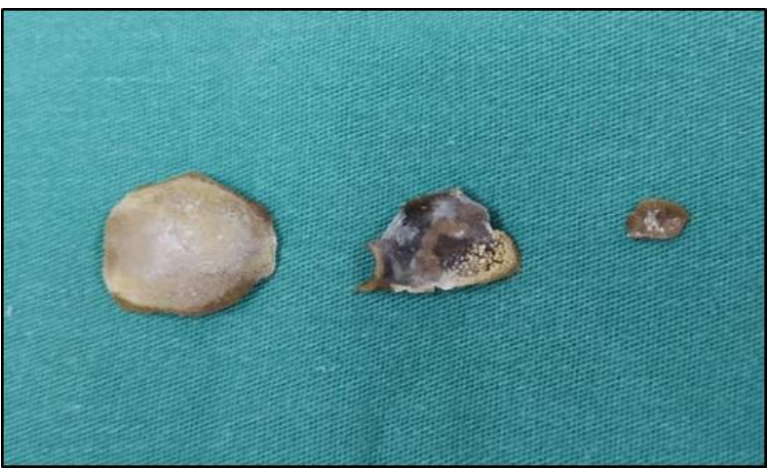

Figura 3. Estruturas ósseas removidas do canal vaginal em evidência.

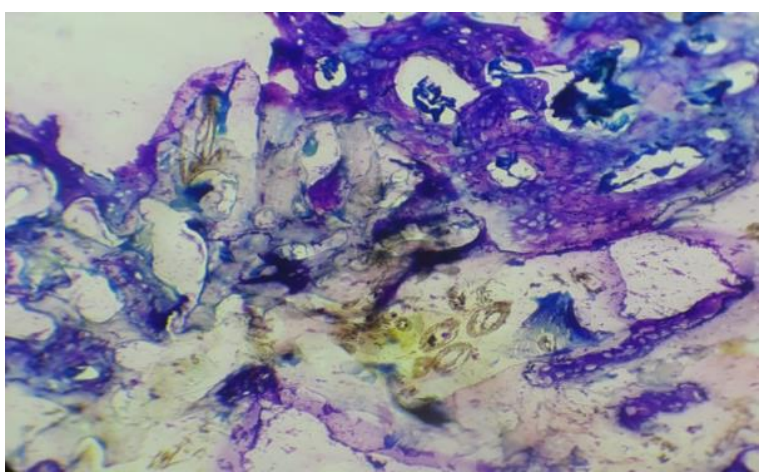

Figura 4. Matriz óssea mineralizada associada a estrutura tecidual com morfologia de pele contendo restos de folículos pilosos e neles queratina (Obj de 40x).

\section{Discussão}

A execução da anamnese completa e exame físico geral proporciona referências importantes para execução do exame ginecológico externo e interno, no qual se embasa na inspeção da vulva, toque vaginal e vaginoscopia, além de exames complementares, nos quais são significativos para o diagnóstico e tratamento de patologias reprodutivas. Em relação a cadelas e gatas o toque vaginal é preconizada para analisar a simetria, irregularidade, estreitamento do canal pélvico e para designar se há presença de tumores na vagina (Dalmazzo \& Ferrari, 2019).

Segundo Kolber (2006) a radiologia é de suma importância para a medicina veterinária e vem evoluindo no auxílio das enfermidades que acometem pequenos e grandes animais. Como observado no caso relatado, onde o animal apresentava estruturas radiopacas no canal vaginal, equivalendo com o exame histopatológico que revelou presença de matriz óssea mineralizada associada à estrutura tecidual com morfologia de pele contendo restos de folículos pilosos e neles queratina, sendo sugestivo de maceração fetal, que é caracterizado pelo processo séptico de destruição fetal retido no 
útero, com amolecimento e liqueficação dos tecidos moles fetais levando a uma esqueletização, considerado assim uma emergência geniturinária (Toniollo \& Vicente, 2003).

Devido à presença de estrutura óssea e infecção do trato urinário apresentado pela paciente, resultou-se em uma inflamação vaginal, com secreção mucopurulenta, caracterizada como vaginite. Para Nelson \& Couto (2015) existem inúmeras afecções do sistema reprodutor dentre elas vaginite. Essa enfermidade se caracteriza pela inflamação da vagina, decorrente de doenças primarias, infecções do trato urinário, presença de corpos estranhos, entre outros fatores. Segundo Duncan \& Prasse (1982), McGavin \& Zachay (2013) e Jones et al.(2000) essa enfermidade, não possui distinção de idade ou raça e pode se desenvolver em cadelas castradas, como do presente caso.

Kahn et al. (2005) afirmam que, a existência de secreção vulvar é um dos sinais clínicos habituais desta enfermidade, podendo variar de mucoide a mucopurulenta, podendo apresentar também sinais clínicos como polaquiúria e lambedura da vulva. A paciente em questão teve como alterações clínicas, secreção vulvar mucopurulenta, polaquiúria e lambedura da vulva, corroborando com a literatura.

No exame complementar ultrassonográfico constatou-se a presença de cistite ascendente em função da vaginite. Este exame possibilita a detecção de várias afecções e anormalidades do trato urinário inferior, que se tornou assim, útil para realização de um tratamento precoce e eficaz (Léveillé, 1998). Conforme Nelson \& Couto (2015) as doenças que acometem o trato urinário inferior são comuns em cães domésticos, nos quais as fêmeas castradas estão perante o maior risco de infecção visto que a propagação de bactérias gram-negativas e gram-positivas se adentram pela vagina e uretra e por via ascendente provocam infecção na vesícula urinaria.

Este relato chama atenção pelo fato da paciente ser castrada há mais de 5 anos e possuir estruturas ósseas fetais em seu canal vaginal, vindo a apresentar sinais clínicos característicos de anormalidades reprodutivas após alguns anos do procedimento. Segundo Johnston et al. (2001), um animal castrado, quando apresenta vaginite, pode ter como causas uma soma de diferentes patologias, incluindo corpo estranho urogenital.

A função primordial do trato urinário inferior é a armazenagem e liberação controlada e periódica da urina, para certificação e localização da patologia do trato urinário é fundamental levar em consideração as informações adquiridas na anamnese exame físico, e dos exames complementares, assim como, no caso relatados o exame físico e complementar foram de suma importância para diagnostico das afecções que estavam acometendo a paciente (Barsanti, 2006; $\underline{\text { Stephen J Ettinger et al., }}$ 2002).

Para o diagnóstico definitivo seria necessário submeter o animal a diversos exames como: cultura e antibiograma de urina, ultrassonografia do trato urinário, radiografia, hemograma completo e exame bioquímico (ureia e creatinina), que são de importância para o diagnóstico de infecção do trato urinário (Monteiro \& Pereira, 2012). Segundo Hartmann \& Baneth (2006) o achado mais relevante no hemograma, quando se tem infecção urinaria, é leucocitose por neutrofilia com ou sem desvio a esquerda. Este um parâmetro a ser considerado quando há suspeita de infecção do trato urinário.

O exame ultrassonográfico, é um mecanismo de diagnóstico por imagem favorável para verificação da vesícula urinária, pois o mesmo facilita a avaliação anatômica e se há presença de patologias com ou sem utilização de contrastes, sendo considerado um órgão de fácil acesso (Gallatti \& Iwasaki, 2004; Lang, 2006). Quando há presença de cistite, assim como diagnosticada na paciente do presente caso, a vesícula urinária apresenta alteração na sua simetria e na espessura da parede principalmente na porção cranial (Lang, 2006).

O teste de cultura e antibiograma da urina é indicado por se tratar de um método de diagnóstico com padrão ouro para infecções do trato urinário, e que irá auxiliar na identificação do agente causador da enfermidade, com propósito de escolher o antibiótico ideal, para que assim, alcance maior eficácia no tratamento (Litster et al., 2011). Deve-se considerar que as bactérias frequentemente isoladas na urina de cães com infecção do trato urinário são gram-negativas como Escherichia coli, Proteus mirabilis, Klebsiella pneumoniae, Pseudomonas aeruginosa e Enterobacter spp. e também gram-positivas como Streptococcus spp. e Staphylococcus spp. (Barsanti, 2006). Para o tratamento da infecção é recomendado o uso de antibióticos, com objetivo de inibir o crescimento bacteriano e sua 
proliferação (Hillier et al., 2014). No caso relatado, o antibiótico de escolha para o tratamento foi da classe das fluorquinolonas. Essa é denominada como antimicrobiano bactericida de amplo espectro, ou seja, com ação em bactérias gram-positivas e gram-negativas empregada no combate de várias infecções, principalmente do sistema urinário (Spinosa et al., 2006).

\section{Conclusão}

Corpos estranhos vaginais são o raro motivador de descarga vaginal em cães. Desta forma, pode ser um problema desafiante no momento do diagnóstico. O exame físico minucioso, associado a exames complementares como os de imagem são de extrema importância para a confirmação do diagnóstico. Neste relato a presença estrutura óssea no canal vaginal constitui um diagnóstico diferencial para a descarga vaginal e cistite recorrente em uma cadela castrada.

A conduta em casos de estruturas ósseas vaginais exige a remoção dos mesmos, visto que pode haver complicações secundárias à sua permanência no local, necessitando ainda de abordagem terapêutica medicamentosa associada para a completa e definitiva resolução do quadro clínico do paciente.

\section{Referências}

Barsanti, J. A. (2006). Genitourinary infecctions. In C. E. Greene (Ed.), Inffections diseases of dogs and cats (3th ed., Vol. 1, pp. 935-961). Saunders.

Dalmazzo, A., \& Ferrari, S. (2019). Exame do sistema genital dos animais. Brazilian Journal of Natural Sciences, 2(3), 202-209. DOI: https://doi.org/10.31415/bjns.v2i3.71

Duncan, J. R., \& Prasse, K. W. (1982). Patologia clínica veterinária. UFSM.

Ettinger, Stephen J, Fedlman, E. C., \& Taibo, R. A. (2002). Tratado de medicina interna veterinaria: enfermedades del perro y el gato. Manole.

Gallatti, L. B., \& Iwasaki, M. (2004). Estudo comparativo entre as técnicas de ultra-sonografia e cistografia positiva para detecção de alterações vesicais em cães. Brazilian Journal of Veterinary Research and Animal Science, 41(1), 40-46. DOI: https://doi.org/10.1590/s1413$\underline{95962004000100007}$

Hartmann, K., \& Baneth, G. (2006). Infectious diseases of the dog and cat. In C. E. Greene (Ed.), Hepatozzon canis Infection (3a ed., Vol. 1). Elsevier Health Sciences.

Hillier, A., Lloyd, D. H., Weese, J. S., Blondeau, J. M., Boothe, D., Breitschwerdt, E., Guardabassi, L., Papich, M. G., Rankin, S., \& Turnidge, J. D. (2014). Guidelines for the diagnosis and antimicrobial therapy of canine superficial bacterial folliculitis (A ntimicrobial $G$ uidelines $W$ orking $\mathrm{G}$ roup of the I nternational $\mathrm{S}$ ociety for $\mathrm{C}$ ompanion A nimal I nfectious $\mathrm{D}$ iseases). Veterinary Dermatology, 25(3), 163-e43. DOI: https://doi.org/10.1111/vde.12118

Johnston, S. D., Kustritz, M. V. R., \& Olson, P. N. S. (2001). Disorders of the canine vagina, vestibule, and vulva. Canine and Feline Theriogenology, 1, 225-242.

Jones, T. C., D., H. R., \& King, N. W. (2000). Patologia veterinária. Manole.

Kahn, C. M., Line, S., \& Aiello, S. E. (2005). The merck veterinary manual. Merck \& Co. Whitehouse Station.

Klein, M. K. (2007). Tumors of the female reproductive system. In S. J. Wihtrow \& E. G. Macewem (Eds.), Small animal clinical oncology (pp. 610-618). Saunders.

Kolber, A. I. (2006). Radiologia em Medicina Veterinária. In A. I. Nobrega (Ed.), Tecnologia Radiológica e Diagnóstico por Imagem (pp. 123-156). Difusão.

Lang, J. (2006). Urinary tract. In P. Mannion (Ed.), Diagnostic ultrasound in small animal practice. Blackwell Publishing.

Léveillé, R. (1998). Ultrasonography of urinary bladder disorders. Veterinary Clinics: Small Animal Practice, 28(4), 799-821. DOI: https://doi.org/10.1016/s0195-5616(98)50079-4 
Litster, A., Thompson, M., Moss, S., \& Trott, D. (2011). Feline bacterial urinary tract infections: An update on an evolving clinical problem. The Veterinary Journal, 187(1), 18-22. DOI: https://doi.org/10.1016/j.tvjl.2009.12.006

McGavin, D., \& Zachay, J. F. (2013). Bases da patologia em veterinária. Elsevier Brasil.

Monteiro, M. V. M. A., \& Pereira, H. (2012). Caracterização das infecções do trato urinário de origem microbiana-casuística de um hospital veterinário em Lisboa. Revista Lusofona de Ciências e Medicina Veterinária, 1, 1-23.

Nelson, R. W., \& Couto, C. G. (2015). Medicina interna de pequenos animais (Issue 1). Elsevier Editora.

Purswell, B. J. (2004). Vaginal disorders. In S. J. Ettinger \& E. C. Feldman (Eds.), Textbook of veterinary internal medicine. Elsevier Saunders.

Sapierzyński, R., Malicka, E., Bielecki, W., Krawiec, M., Osińska, B., Sendecka, H., \& SobczakFilipiak, M. (2007). Tumors of the urogenital system in dogs and cats. Retrospective review of 138 cases. Polish Journal of Veterinary Sciences, 10(2), 97-103.

Soderberg, S. F. (1986). Vaginal disorders. Veterinary Clinics of North America: Small Animal Practice, 16(3), 543-559. DOI: https://doi.org/10.1016/s0195-5616(86)50060-7

Spinosa, H. S. de S., Górniak, S. L., \& Bernardi, M. M. (2006). Farmacologia aplicada à medicina veterinária. Koogan Guanabara.

Tilley, L. P., Smith, J. R., \& Francis, W. K. (2008). Consulta veterinária em 5 minutos: Espécies canina e felina. Editora Manole.

Toniollo, G. H., \& Vicente, W. R. R. (2003). Manual de obstetrícia veterinária. Varela.

Zachary, J. F., McGavin, D., \& McGavin, M. D. (2012). Bases da patologia em veterinária. Elsevier Brasil.

Histórico do artigo:

Recebido: 27 de março de 2021.

Aprovado: 3 de maio de 2021 .
Licenciamento: Este artigo é publicado na modalidade Acesso Aberto sob a licença Creative Commons Atribuição 4.0 (CC-BY 4.0), a qual permite uso irrestrito, distribuição, reprodução em qualquer meio, desde que o autor e a fonte sejam devidamente creditados. 